\title{
Pascal Ughetto, Faire face aux exigences du travail contemporain. Conditions du travail et management
}

Anne-Marie Waser

\section{OpenEdition}

\section{Journals}

Édition électronique

URL : http://journals.openedition.org/travailemploi/2001

DOI : 10.4000/travailemploi.2001

ISSN : 1775-416X

Éditeur

DARES - Ministère du Travail

\section{Édition imprimée}

Date de publication : 15 juin 2008

Pagination : 87-88

ISSN : 0224-4365

\section{Référence électronique}

Anne-Marie Waser, «Pascal Ughetto, Faire face aux exigences du travail contemporain. Conditions du travail et management », Travail et Emploi [En ligne], 114 | avril-juin 2008, mis en ligne le 05 novembre 2010, consulté le 08 avril 2021. URL : http://journals.openedition.org/travailemploi/2001 ; DOI :

https://doi.org/10.4000/travailemploi.2001 


\section{Faire face aux exigences du travail contemporain. Conditions du travail et management}

\section{Pascal UGHETTO}

Lyon, éditions de l'ANACT, 2007

\section{Lu par Anne-Marie Waser (*)}

Sauf à y prendre garde, cet ouvrage de 157 pages peut passer inaperçu du lecteur universitaire qui pourrait imaginer qu'il est principalement destiné aux acteurs d'entreprises, alors que, en réalité, il est bien fait aussi pour faire évoluer la réflexion et les outils d'analyse propre à l'univers académique.

L'originalité de la question de départ de Pascal Ughetto - quel sort est-on obligé de faire aux travailleurs pour que les produits sortent avec toutes les exigences qui leur sont dorénavant assignées? - permet d'aborder, sans détour, l'épreuve de réalité à laquelle se confrontent les actifs quotidiennement. Or, force est de constater que l'activité de travail est un impensé dans les entreprises comme dans les administrations.

Pour avancer sur cette question de départ, l'auteur propose de parler de l'exigence du travail contemporain afin de se départir des représentations usuelles - très largement juridiques - des conditions de travail. Entre les conditions de travail et les conditions $d u$ travail, il y a la différence entre, le fait d'être employé sur un poste ou une fonction, et le fait de produire, de réaliser une tâche. Le point aveugle des relations professionnelles instituées est de ne pas faire entrer tout ce qui concerne les conditions dans lesquelles se trouvent les travailleurs pour produire. Pourtant, selon l'auteur, les conditions du travail mériteraient d'être revalorisées comme problème public.

Le virage sémantique proposé par Pascal Ughetto permet de sortir d'une opposition stérile entre performance et maintien du statut. Partir des exigences qu'incorporent les produits contemporains (promesses du marketing, règles sanitaires, coordination de fournisseurs multiples, etc.) permet d'aborder la question du travail de la façon dont elle se pose pour celui qui le réalise, qu'il soit cadre ou non cadre. Le travail est le niveau où l'on «encaisse» tous ces impératifs, où l'on est forcé de les assembler.

Le premier chapitre ouvre sur la mise sous tension du travail et sur ses effets bien connus et mesurés par les enquêtes. Mais, si le thermomètre monte, ni les managers, ni les syndicalistes ne parviennent à établir le diagnostic ou à faire évoluer la situation.

(*) Centre d'études de l'emploi.
Les grandes organisations promettent à leurs clients ou usagers le respect de hauts standards d'exigence. Une promesse dont l'auteur montre bien qu'elle n'a d'égal que l'ampleur de la tâche à réaliser. En prenant l'exemple de l'évolution des produits offerts par les boulangeries de quartier; une variété de pain disponible à toute heure jamais atteinte, des fournées multipliées, des types de cuisson différente, Pascal Ughetto convainc le lecteur que l'exigence est du côté du consommateur, qu'elle est positive, permanente et sans doute irréversible. Mais cette exigence a des effets importants sur l'organisation du travail des boulangers et sur la densification des tâches. Elle transforme le métier en le rendant certes, plus intéressant, mais aussi plus complexe par la multiplication des acteurs (fournisseurs) qui amène de l'incertitude, l'ajout d'activités (marketing, communication) et des délais à tenir.

Dans le deuxième chapitre, Pascal Ughetto montre qu'un des effets de la montée en puissance des outils de gestion des ressources humaines et des doctrines qui les accompagnent, est l'alimentation des dynamiques de l'incompréhension entre les opérationnels et le management. L'auteur rappelle que, historiquement, la «fonction personnel», souvent centralisée, s'est constituée en privant les contremaîtres de leurs prérogatives de gestion de la main-d'œuvre. Or cette façon de gérer de loin le personnel avec des consignes hiérarchiques prodiguées en toute généralité ne peut en aucun cas tenir avec les autres formes de prescription, de jugement et d'évaluation auxquelles le travailleur est confronté dans son quotidien.

Mais prendre au sérieux la gestion, et plus particulièrement la façon dont elle organise la montée en capacité de sortir les produits, requiert une organisation de la capacité d'agir. Cette capacité ne signifie pas le contrôle des conduites. L'auteur rappelle que les travailleurs sont des sujets irréductiblement libres, qui ont une subjectivité, une économie psychique, et qui ne pourront pas être entièrement subordonnés aux impératifs de l'employeur. Ils construisent un rapport à leur travail qui évolue, ils donnent un sens à leur travail; ils réévaluent en permanence leur engagement dans le travail, etc.

Le monde du travail contemporain manque d'acteurs capables de donner une audience aux problèmes du travail nous dit Pascal Ughetto. Les responsables de ressources humaines ainsi que les responsables syndicaux a priori les mieux placés pour faire remonter en haut les problèmes rencontrés par les opérationnels restent ficelés dans leurs rôles confortés par des théories qui leur rendent difficile l'analyse des problèmes concrets. Pour faire évoluer cette situation l'auteur propose de rééquilibrer le pouvoir en faveur des directions «métier» et des cadres opérationnels (responsables de sites, chefs d'unité de production) qui, face à la puissance des logiques marketing et de communication, ont perdu le fil de la logique de production.

Autoriser à dire qu'on a du mal à faire, qu'on n'est pas facilement performant, est le pas que ne 
parviennent généralement pas à franchir les entreprises en raison du leitmotiv de la «performance». Or, souligne l'auteur, pour être performant, il faut parler de ce qui ne va pas. Ainsi, c'est tout une démarche et un changement de rapport de force qu'il faudrait instaurer dans les entreprises. Il faut intéresser les entreprises à la reconnaissance des problèmes du travail comme fondement de l'innovation. Prendre le travail et les expériences des travailleurs au sérieux. Autoriser ceux qui produisent à parler de leur travail. Donner un statut à l'expression des opérationnels.

Sans faire la genèse des conditions du travail, Pascal Ughetto note qu'elles ont été pensées indépendamment de l'organisation du travail. S'il était possible de le penser hier, cela ne l'est plus aujourd'hui. En effet, la question des conditions du travail ne peut plus être traitée indépendamment de celle des compétences individuelle et collective, de l'organisation de l'activité de travail, de son management, ou encore de celle de l'engagement dans le travail.

Dans la troisième partie Pascal Ughetto pose la question de quelle(s) condition(s) pour le travail? Face à l'exigence du travail, les salariés sont-ils managés ou abandonnés? Il souligne les contradictions de la montée en autonomie qui semblent, paradoxalement, se retourner contre les salariés dans les services notamment qui se plaignent: «Ce n'est pas managé». Cette autonomie serait une réponse au problème de la productivité dans l'économie des services. L'instabilité des services par rapport aux produits et la forte dépendance des services vis-àvis de l'environnement fausse les calculs classiques de productivité. Du coup, c'est l'activité qui est mise sous tension de comptes à rendre; d'indicateurs de performance, de procédures, de protocoles impératifs à respecter, etc. alors que le besoin des salariés est de voir leur activité de travail organisée. D'où une pénibilité, des souffrances qui indiquent moins le contrôle exercé par les employeurs que leur manque de maîtrise de processus d'organisation de l'activité.

Cependant, l'organisation de l'activité, précise Pascal Ughetto, est un investissement auquel les employeurs ne consentent pas aisément. En effet, ceux qui ont adopté, par exemple, une gestion participative des réformes connaissent des bilans mitigés. La piste que suggère l'auteur pour faire évoluer la question du management et des conditions du travail est celle de la représentation du travail et du travailleur car les conditions du travail contemporain ont bien fondamentalement à voir avec le management et plus particulièrement avec le management de l'engagement. Cette question, déjà travaillée en sociologie du travail, met en évidence la difficulté de définir et cerner la tâche dans les situations de relation de service.

En conclusion, ce livre, riche de situations concrètes qui ébranlent les théoriques implicites du travail, amène à interroger les conditions dans lesquelles on fait travailler les gens. Il invite à tenir ensemble deux éléments indissociables: 1) les dispositifs gestionnaires qui ont des effets sur l'activité concrète; 2) la condition faite au travail (la façon dont on reconnaît la contribution du travail par rapport à la planification des actions édictée par l'employeur) et aux travailleurs (comment les gens sont traités).

Cet ouvrage original peut être salué pour son efficacité, tant au niveau de la langue utilisée, que des formes de démonstration qui rendent les résultats de la recherche accessibles aux gens d'entreprises sans rien sacrifier aux exigences de sérieux scientifique. Par un travail minutieux qui amène à préciser la tâche, le rôle de chacun des acteurs dans les processus de production en constante mutation, Pascal Ughetto permet ainsi de desserrer l'étau dans lequel beaucoup de travaux des dernières années ont eu tendance à nous enfermer: travaux sur l'intensification du travail, la montée de la domination, qui ont eu tendance à nous faire un tableau accablant des évolutions du travail et du sort infligé aux salariés.

L'approche novatrice et réussie, car convaincante, de l'auteur repose d'abord sur le recours à la clinique du travail développée par Yves Clot. La mobilisation de cette approche permet à l'auteur d'éviter de s'enfermer dans une sociologie des rapports de domination et laisse davantage de place à un sujet qui ne se réduit pas à faire preuve d'une capacité de résistance, voire de participation, à la domination. D'autre part, l'approche du livre interpelle le lecteur dans son vécu (travailleur subordonné-manager, consommateur, usager, etc.), car le parti pris d'enquête qui est de prendre au sérieux les acteurs du management, de les incarner (non pas des managers abstraits mais, souvent, des cadres, voire des cadres opérationnels, distingués d'autres cadres, ceux des ressources humaines, par exemple), de considérer leurs contraintes, le travail qu'ils réalisent, etc.

$\mathrm{Si}$ à la première lecture, on a le sentiment que ce sont des choses relativement simples qui sont dites, cela n'a été possible que par un travail théorique, fait en back office, pour réunir et marier des perspectives théoriques et des ensembles de travaux généralement distincts : la sociologie du travail renouvelée par l'approche pragmatique de J.-M. Weller (interactionnisme de la relation de service), la clinique du travail (Y. Clot), la sociologie des outils de gestion (M. Berry, J.-C. Moisdon) et des éléments d'économie industrielle. C'est au prix d'une maîtrise des concepts et des problématiques appartenant à différentes disciplines (sociologie, psychopathologie du travail, ergonomie, gestion, économie) que Pascal Ughetto parvient à créer un point de vue propice à tenir dans une même analyse le travail, le travailleur, les outils, les dispositifs et les contraintes sans dissonance, ni simplification des situations, ni omission des temporalités.

La limite de cet ouvrage qui vise une large accessibilité est celle précisément des soubassements théoriques. Le lecteur qui ne maîtrise pas toutes ces problématiques aimerait les voir davantage mises en œuvre et travaillées. 